\title{
NURSE-SY-1-3
}

\section{Complications after liver surgery}

\author{
Bong-Wan KIM
}

Department of Liver Transplantation and Hepatobiliary Surgery, Ajou University Hospital, Suwon, Korea

Lecture: Two decades ago, mortality rates after hepatectomy had been reported approximately $9 \%$ to $20 \%$ or more. Advances in appropriate patient selection, operative and anesthetic techniques, and perioperative care have reduced the risk of mortality less than $3 \%$. In contrast, morbidity rate still remains high, ranges from $20 \%$ to $56 \%$, depending on the patient condition, the extent of resection, the remnant liver functional reserve, the underlying liver disease, and the surgical skill. Operation on the liver is especially challenging because of its unique anatomic architecture and its vital functions. Various post-hepatectomy complications could occur such as post-hepatectomy liver failure, postoperative hemorrhage, bile leakage, ascites, pleural effusion, pulmonary atelectasis or infection, venous thromboembolism, and surgical site infection. These problems are closely related to preoperative evaluation and preparation, surgical and anesthetic technique, and postoperative monitoring and management. Currently, the liver surgeons are increasingly performing complex operations with more extended resections and more repeat hepatectomies on an aging population with multiple comorbidities and heavily pretreated or hepatitis-damaged livers. A well-designed, individualized management approach based on patient's overall condition and liver function is vital to reduce post-hepatectomy complications and to achieve optimal outcomes. This presentation would introduce type of complications after liver resection and its management. 